\title{
A Randomized Comparison of a Parker Endotracheal Tube and a Standard Tube Oriented $90^{\circ}$ Counterclockwise
}

\author{
Wade A. Weigel, Thomas C. Dean \\ Department of Anesthesiology, Virginia Mason Medical Center, Seattle, USA. \\ Email: wade.weigel@vmmc.org, dean.t@ghc.org
}

Received August $10^{\text {th }}, 2012$; revised September 11 $1^{\text {th }}$,2012; accepted September 21 $1^{\text {st }}, 2012$

\begin{abstract}
Purpose: During oral fiberoptic intubation, advancement of an endotracheal tube (ETT) into the trachea is occasionally impeded by laryngeal structures. The curved flex tip Parker ETT has been shown to improve the likelihood of successful advancement as opposed to a standard ETT that is advanced in neutral orientation. However, a Parker tube has not been compared to a standard ETT oriented $90^{\circ}$ counterclockwise from the neutral position. We hypothesize that fiberoptically-guided advancement of an ETT into the trachea will be more successful when using a Parker tube than a $90^{\circ}$ counterclockwise-oriented standard ETT. Methods: This unblinded, randomized controlled trial compares the rate of successful advancement of a fiberoptically-guided endotracheal tube into the trachea. Two groups of randomly assigned patients with non-difficult airways are compared: a Parker flex-tip tube (Parker Group; $n=57$ ) versus a standard ETT oriented $90^{\circ}$ counterclockwise (Standard Group; $n=58$ ). Our primary outcome is the first pass success rate of advanceing the ETT into the trachea. Results: First pass success occurred in 48 of 57 (84\%) patients in the Parker Group vs. 39 of $58(67 \%)$ of patients in the Standard Group ( $p=0.0497)$. Conclusion: When advancing an ETT over an oral fiberoptic scope and into the trachea, a Parker curved flex tip ETT is statistically more likely to be placed successfully on the first pass than is a standard ETT oriented $90^{\circ}$ counterclockwise.
\end{abstract}

Keywords: Endotracheal; Intubation; Parker; Mallinckrodt; Fiberoptic

\section{Introduction}

When performing fiberoptic-guided oral endotracheal intubation, studies report that a standard bevel endotracheal tube (ETT) in neutral orientation, i.e. rotated neither clockwise nor counterclockwise, successfully passes into the trachea on $11 \%$ to $47 \%$ of the first attempts [1-3]. Failure of the ETT to advance into the trachea typically results when its tip impinges on laryngeal structures such as the arytenoid cartilage or interarytenoid soft tissue $[3,4]$. Attempting to manipulate the ETT beyond these obstructing laryngeal structures by rotation, or withdrawal and reinsertion, can lead to oxygen desaturation, hypopharyngeal trauma, vocal cord paralysis, or esophageal intubation $[5,6]$. The Parker ETT (Figure 1) was designed to facilitate passage into the glottic opening by providing a soft, curved, flexible tip that is oriented $90^{\circ}$ counterclockwise from the neutral position.

The first pass success rate for advancing a Parker ETT into the trachea during oral fiberoptic-guided intubation is significantly higher compared to a standard endotracheal tube in neutral orientation (Figure 1) $(71 \%$ vs $11 \%$, respectively) [7]. However, this previous study was criticized because the compared standard ETT was not rotated $90^{\circ}$ counterclockwise to match the same bevel orientation as that of the Parker tube [8,9]. The assertion is that if the tips were oriented the same there would be no benefit to the Parker endotracheal tubes soft tip and curved design.

Our study was designed to test whether a Parker tube is superior to a similarly oriented standard ETT for facilitating oral fiberoptic intubation. We hypothesize that the curved tip Parker tube will result in a higher frequency of successful first pass advancements of the ETT into the tracheal than will a standard ETT in the same orientation.

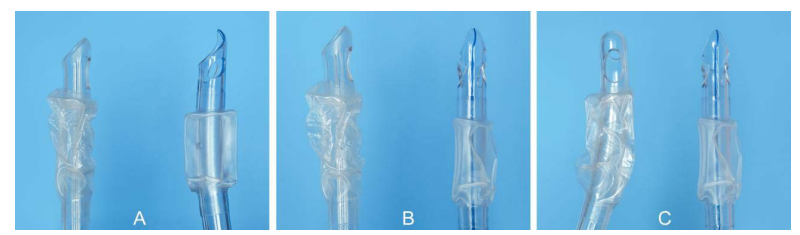

Figure 1. Anterior-Posterior views of the studied endotracheal tubes. (A) A view of the standard 279 (left) and Parker ETT (right) to show the differences in tip design; (B) Standard orientation of 280 the ETT tips; (C) Orientation of the ETT tips for this study. 


\section{Methods}

This study was approved by the Institutional Review Board of the Benaroya Research Institute at Virginia Mason Medical Center. The primary or sub-investigator explained the study and procured the written informed consent of subjects on the day of surgery. Adult patients ( $>18$ years of age) undergoing elective surgery requiring general anesthesia and endotracheal intubation, who had no risk factors for a difficult airway and were ASA class III or less, were eligible for enrollment. Risk factors for difficulty with intubation were considered to include a history of prior difficult intubation, Malampati class $>3$, thyromental distance $<4 \mathrm{~cm}$, and a cervical range of motion $<80$ degrees.

Patients were randomized to receive either a Mallinkrodt or Parker Flex-Tip ETT (Parker Medical, Englewood, Colorado) based upon assignment from a computerized random number generator. This assignment was printed on a piece of paper and placed into a sealed, numbered envelope. The number reflected the order with which the envelopes would be opened, and the patients were assigned a number based upon their order of entry into the study. After obtaining informed written consent, the patient was assigned the next number in sequence and the corresponding envelope was opened, revealing the allocation to either Parker or Mallinkrodt ETT.

We compared a standard $8.0 \mathrm{~mm}$ inner diameter (ID) Mallinckrodt (Mallinckrodt Inc, Hazelwood, Missouri) ETT with a $8.0 \mathrm{~mm}$ ID Parker Flex-Tip (Parker Medical, Englewood, Colorado) ETT. An 8.0 mm ID ETT size was chosen to emphasize the difference in tube tip design between the Parker and Mallinkrodt tubes, as opposed to endotracheal tube size. Patients were randomized to receive either a Mallinkrodt (standard group) or Parker tube (Parker group). No efforts were made to blind the operators performing either the fiberoptic portion of the procedure or the advancement of the endotracheal tube over the fiber bundle because the steps for advancement were different for the two types of ETT. Prior studies have used plastic bags placed over the endotracheal tube as a measure to blind the operators. We considered this method of blinding, but regard it as cumbersome and potentially dangerous, as visualization of the endotracheal tube during advancement is important for the prevention of pharyngeal trauma.

The patients were taken to the operating room, where standard monitors were placed before general anesthesia was induced. Muscle relaxation was used in all inductions; however, the agents used were left to the discretion of the attending anesthesiologist. Muscle relaxation was not routinely evaluated with nerve stimulation prior to intubation attempts. The mouth was opened and the tongue retracted anteriorly via gauze retraction by a primary investigator (WAW or TCD). Bimanual jaw thrust was applied in all intubations. An oral fiberoptic in tubating airway (e.g., Ovassapian intubating airway) was not used for any of the study participants. The fiberoptic scope (4.1 mm outer diameter, Olympus LF-GP or Olympus LF-V, Olympus Optical, Tokyo, Japan) was then introduced orally and advanced into the trachea by the anesthesiologist performing the anesthetic. Once the fiberscope was inside the trachea, a primary investigator then advanced the endotracheal tube over the fiberscope into the trachea. Correct placement was verified by fiberoptic visualization of the carina. The Parker ETTs were oriented with the curve of the ETT coincident with the curvature of the hypophaynx. Mallinckrodt tubes were initially oriented in the same manner, but upon entering the hypopharynx (tip just past the base of the tongue) the tubes were rotated 90 degrees counterclockwise prior to advancing through the glottic opening.

If either ETT did not pass on the first attempt, it was retracted $5 \mathrm{~cm}$ and readvanced without changing orientation. If this was not successful, the ETT was again retracted $5 \mathrm{~cm}$ and rotated independent of the fiberscope 90 degrees clockwise and readvanced. If the ETT did not advance easily after the second repositioning (3rd attempt overall), then the fiberoptic scope was removed and intubation performed using direct laryngoscopy.

The overall success rate includes first pass success, second pass success with no change in orientation, and third pass success after 90 degree counterclockwise rotation.

\section{Statistical Analysis}

Prospective power analysis was performed assuming the data would be analyzed by comparing the proportion of successful intubations on first attempt, $\alpha=0.05, \beta=0.8$, and two-tailed analysis Based on these assumptions, power analysis using Statmate software (Graphpad Software, La Jolla, California) indicated that 125 subjects would be able to detect a difference of $15 \%$ between groups. The study of 70 patients by Kristensen et al. [7] detected a difference of $60 \%$ between the groups. Joo et al. [10] studied 111 patients and found a difference of $7 \%$, which was not statistically significant. Since we planned to turn the Mallinckrodt ETT 90 degrees counterclockwise to improve first pass success, we expected a smaller difference than found in the Kristensen study. We elected to use only $8.0 \mathrm{~mm}$ OD ETTs so we expected to find a greater difference than Joo et al., who used 7.0, 7.5, and $8.0 \mathrm{~mm}$ ID ETTs.

The Fisher exact test was used to compare rates of both first-pass and overall success rates between the Parker and Mallinckrodt tubes. The Mann-Whitney test and $t$ tests were used to compare demographic features of the individual sample populations. 


\section{Results}

One-hundred and twenty patients were recruited and consented for inclusion in the study. Final analysis included 115 patients (Parker Group $n=57$, Standard Group $n=58)$. The remaining 5 patients were excluded for incomplete data collection or protocol violation (3 patients in the Parker group and 2 patients in the Standard group). There were no statistical demographic differences in patient characteristics between study groups (Table 1).

Patients were recruited between October 2008 and March 2009. The study was terminated after reaching the target recruitment number.

There was a statistically significant difference in first pass success rate in favor of the Parker ETT (Table 2). Forty eight of the fifty seven patients intubated using Parker tubes were successfully intubated on the first-pass (84\%). In the standard ETT group, 39 of the 58 patients were successfully intubated on the first attempt (67\%, p $=0.0497$ ). There was no statistically significant difference in terms of second pass, third pass, or overall intubation rates (Table 2 ).

Table 1. Patient characteristics.

\begin{tabular}{ccc}
\hline $\begin{array}{c}\text { Demographics/Airway } \\
\text { Characteristics }\end{array}$ & $\begin{array}{c}\text { Mallinkrodt } \\
n=58\end{array}$ & $\begin{array}{c}\text { Parker } \\
n=57\end{array}$ \\
\hline Age, yrs (mean \pm SD) & $56 \pm 13$ & $56 \pm 14$ \\
BMI, $\mathrm{kg}^{2} / \mathrm{m}$ (mean \pm SD) & $27 \pm 5$ & $28 \pm 5$ \\
Male/female & $67.2 \%$ & $57.9 \%$ \\
ASA status (median, quartiles) & $2.2-3$ & $2.2-2$ \\
$\begin{array}{c}\text { Mallampati score } \\
\text { (median, quartiles) }\end{array}$ & $1.1-2$ & $2.1-2$ \\
$\begin{array}{c}\text { Thyromental distance, } \\
\text { cm (mean } \pm \text { SD) }\end{array}$ & $5.6 \pm 0.7$ & $5.7 \pm 0.5$ \\
\hline
\end{tabular}

All differences NS.

Table 2. Success rates.

\begin{tabular}{cccc}
\hline & $\begin{array}{c}\text { Parker } \\
n=57\end{array}$ & $\begin{array}{c}\text { Mallinkrodt } \\
n=58\end{array}$ & p value \\
\hline $\begin{array}{c}\text { First-pass } \\
\text { Success Rate }\end{array}$ & $48 / 57(84 \%)$ & $39 / 58(67 \%)$ & 0.0497 \\
$\begin{array}{c}\text { Second-pass } \\
\text { Success Rate }\end{array}$ & $5 / 9(56 \%)$ & $11 / 19(58 \%)$ & 1.0000 \\
$\begin{array}{c}\text { Third-pass } \\
\text { Success Rate }\end{array}$ & $0 / 4(0 \%)$ & $1 / 8(12.5 \%)$ & 1.0000 \\
Overall Success & & & \\
Rate & & & \\
\hline
\end{tabular}

*Overall success rate includes first pass success, second pass success with no change in orientation, and third pass success after 90 degree clockwise rotation.

\section{Discussion}

No prior study determined first pass success rate when the standard ETT was oriented 90 counterclockwise during the initial attempt. We were able to show a statisticcally significant difference in the first pass success rate ( $p=0.0497)$ when using the Parker ETT (84\%) versus a standard ETT rotated 90 degrees counterclockwise (67\%). This first pass success rate using the Parker ETT is comparable to two other studies that found first pass success rates of $71 \%$ and $91 \%[7,10]$. Lack of blinding may have affected the results; however, given the fact that the type of ETT dictated the need to rotate, we could not have logistically performed adequate blinding. Our study also looked at success rate after initial first pass failure. The success rates after one or two additional attempts at advancements were improved for both types of ETT. The overall success rate for the Parker remained higher (93\%) than for the Mallinckrodt (88\%) but this failed to reach statistical significance $(p=0.5281)$. It appears that with attempts at reinsertion the Parker flex tip design is less advantageous. One argument given for the use of the Parker ETT is that anesthesia providers are, in general, inexperienced with fiberoptic intubations and the maneuvers required for optimizing success [11,12]. From this standpoint, one may infer that clinicians ex- perienced in fiberoptic intubations may have less need to use a flexible tip ETT.

Prior studies have compared Parker ETTs to Mallinckrodt ETTs. One study compared the Parker ETT with a Mallinckrodt ETT turned 90 degrees clockwise (as opposed to counterclockwise) [10]. This study of $111 \mathrm{pa}-$ tients differed from ours in more than just the orientation of the Mallinckrodt ETT. They performed awake fiberoptic intubations in patients with a difficult airway, previous failed intubation, or an unstable cervical spine. The first pass success rate was $91 \%$ using the Parker ETT and $84 \%$ using the Mallinckrodt ETT. This difference did not reach statistical significance $(\mathrm{p}=0.24)$, and thus the authors concluded there was no advantage to using the Parker ETT during awake fiberoptic intubations.

The decision to rotate the Mallinckrodt ETT counterclockwise deserves further discussion. There have been human and manikin studies showing the arytenoids as the main structure of impingement during oral fiberoptic intubation. A counterclockwise 90-degree rotation orients the standard ETT tip farthest away from the arytenoids. The study by Johnson et al. [3] used two fiberoptic scopes during awake oral fiberoptic intubations. The second scope (inserted nasally) recorded the events as the ETT was advanced over the oral fiberoptic scope. In this study, all cases of obstruction were due to impingement on the right arytenoid or interarytenoid tissue. The concept of counterclockwise rotation was explored in 1989 
with a small study showing improved success rate with this maneuver [13]. ETT impingement was also studied using a mannequin model, which also showed that during oral fiberoptic intubation the site of obstruction was the right arytenoid [4]. As a note of caution, it has been suggested that turning the ETT before advancing may lead to "flicking" the fiberoptic scope out of the trachea [14].

Counterclockwise rotation was also used in the Kristensen study that compared the Parker ETT and a standard (Portex) ETT, but the rotation occurred after initial resistance to advancement [7]. As pointed out in two commentaries $[8,9]$, if one were to combine the first pass and second pass success in the Kristensen study (thereby including the counterclockwise rotation maneuver), the success rate of the standard ETT would improve to $68 \%$ (compared to the Parker first pass success rate of $71 \%$ ). This reevaluation of the data led to the presumption that rotating the Mallinckrodt ETT before insertion would allow it to perform as well as the Parker ETT. Our study directly addresses this issue and answered that question.

In our study, we oriented the standard ETT bevel to the same position as the Parker in an attempt to isolate the role that the curved tip plays in bridging the gap between the outer diameter (OD) of the fiberoptic bundle and the inner diameter (ID) of the ETT. This gap has been implicated as a key factor in intubation success rates. One study used different sized fiberoptic scopes (3 $\mathrm{mm}$ OD and $5 \mathrm{~mm}$ OD) and found a higher success rate with the larger fiberoptic scope [15]. Another study looked at different sized ETTs (Mallinckrodt wire reinforced $6 \mathrm{~mm}$ and $8.0 \mathrm{~mm}$ ETTs) over the same size fiberoptic scope $(4 \mathrm{~mm})$. They found a higher first pass success with the smaller ETT [16]. A commentary has also been published that supported this idea by showing a way to bridge the gap by placing a pediatric ETT within an adult ETT [17]. This increased the first pass success rate from $10 \%$ to $100 \%$ in 30 patients. The overarching idea captured by these studies is that the greater distance between the outer diameter of the fiberoptic bundle and the inner diameter of the endotracheal tube the more likely the tip will catch on laryngeal structures. Previous studies comparing Parker and standard ETTs have used various sized ETTs, and hence have not controlled for this important variable. We set the size of the ETT (8.0 $\mathrm{mm}$ ID for all patients) and fiberoptic scope (4.1 mm OD) for a more rigorous evaluation of the influence of tip design. We reasoned that a greater diameter difference would be the situation where a curved, flexible tip (Parker ETT) would confer the greatest advantage.

Our data shows that the Parker ETT is statistically more likely to successfully enter the trachea on first pass during oral fiberoptic intubation. While statistically significant, the clinical significance of this difference is marginal. Subsequent manipulations of either ETT result in an equivalent likelihood of successful intubation.

\section{Acknowledgements}

The authors would like to thank the late Dr. Christopher $M$. Bernards for his invaluable assistance in both the statistical analysis of our results and the editing of our manuscript.

\section{REFERENCES}

[1] H. E. Jones, A. C. Pearce and P. Moore, "Fiberoptic intubation: Influence of Tracheal Tube Tip Design,” Anaesthesia, Vol. 48, 1993, pp. 672-674.

[2] P. Hakala, T. Randell and H. Valli, "Comparison between Tracheal Tubes for Orotracheal Fibreoptic Intubation,” British Journal of Anaesthesia, Vol. 82, No. 1, 1999, pp. 135-136. doi:10.1093/bja/82.1.135

[3] D. M. Johnson, M. Aaron, R. B. Smith, P. Robert and M. A. Maktabi, "Endoscopic Study of Mechanisms of Failure of Endotracheal Tube Advancement into the Trachea during Awake Fiberoptic Orotracheal Intubation," Anesthesiology, Vol. 102, No. 5, 2005, pp. 910-914. doi:10.1097/00000542-200505000-00008

[4] T. Katsnelson, E. A. M. Frost, E. Farcon and P. L. Goldiner, "When the Endotracheal Tube Will Not Pass over the Flexible Fiberoptic Bronchoscope [Letter],” Anesthesiology, Vol. 76, No. 1, 1992, pp. 151-152. doi:10.1097/00000542-199201000-00030

[5] M. A. Maktabi, H. Hoffman, G. Funk and R. P. From, "Laryngeal Trauma during Awake Fiberoptic Intubation," Anesthesia \& Analgesia, Vol. 95, No. 4, 2002, pp. 11121114.

[6] S. Moorthy and S. Dierdorf, "An Unusual Difficulty in Fiberoptic Intubation,” Anesthesiology, Vol. 63, No. 2, 1985, p. 229. doi:10.1097/00000542-198508000-00031

[7] M. S. Kristensen, "The Parker Flex-Tip Tuber versus a Standard Tube for Fiberoptic Orotracheal Intubation, a Randomized Double-Blind Study," Anesthesiology, Vol. 98, No. 2, 2003, pp. 354-358. doi:10.1097/00000542-200302000-00014

[8] A. M. Ho, D. C. Chung and M. K. Karmakar, "Is the Parker Flex-Tip Tube Really Superior to the Standard Tube for Fiberoptic Orotracheal Intubation? [Letter]," Anesthesiology, Vol. 99, No. 5, 2003, p. 1236. doi:10.1097/00000542-200311000-00038

[9] M. Wheeler and R. M. Dsida, "Fiberoptic Intubation: Troubles with the 'Tube' [Letter]," Anesthesiology, Vol. 99, No. 5, 2003, pp. 1236-1237. doi:10.1097/00000542-200311000-00039

[10] H. S. Joo, V. N. Naik and G. L. Savoldelli, "Parker Flex-Tip Are Not Superior to Polyvinylchloride Tracheal Tubes for Awake Fibreoptic Intubations," Canadian Journal of Anaesthesia, Vol. 52, No. 3, 2005, pp. 297-301. doi:10.1007/BF03016067

[11] M. S. Kristensen and J. Moller, “Airway Management Behavior, Experience and Knowledge among Danish Anaesthesiologists-Room for Improvement,” Acta Anaesthesi- 
ologica Scandinavica, Vol. 45, No. 9, 2001, pp. 11811185. doi:10.1034/j.1399-6576.2001.450921.x

[12] M. S. Kristensen, "Is the Parker Flex-Tip Tube Really Superior to the Standard Tube for Fiberoptic Orotracheal Intubation? [Letter-Author Reply]," Anesthesiology, Vol. 99, No. 5, 2003, p. 1237. doi:10.1097/00000542-200311000-00040

[13] D. Schwartz, C. Johnson and J. Roberts, “A Maneuver to Facilitate Flexible Fiberoptic Intubation [Letter]," Anesthesiology, Vol. 71, No. 3, 1989, pp. 470-471. doi:10.1097/00000542-198909000-00038

[14] I. Calder, "When the Endotracheal Tube Will Not Pass over the Flexible Fiberoptic Bronchoscope [Letter]," Anesthesiology, Vol. 77, No. 2, 1992, pp. 398-399. doi:10.1097/00000542-199208000-00036

[15] P. Hakala and T. Randell, "Comparison between Two Fiberscopes with Different Diameter Insertion Cords for Fiberoptic Intubation,” Anaesthesia, Vol. 50, No. 8, 1995, pp. 735-737. doi:10.1111/j.1365-2044.1995.tb06108.x

[16] K. Koga, T. Asai, I. P. Latto and R. S. Vaughan, "Effect of the Size of a Tracheal Tub and the Efficacy of the Use of the Laryngeal Mask for Fiberscope-Aided Tracheal Intubation,” Anaesthesia, Vol. 52, No. 2, 1997, pp. 131-135. doi:10.1111/j.1365-2044.1997.31-az0058.x

[17] N. J. Marsh, "Easier Fiberoptic Intubations," Anesthesiology, Vol. 76, 1992, pp. 860-861. doi:10.1097/00000542-199205000-00031 